Editorial

\title{
Theranostic Biocolloids: Soft Matter Colloids for Imaging and Therapy
}

\author{
Mark Borden ${ }^{1 凶}$, Kaushal Rege ${ }^{2}$ \\ 1. Department of Mechanical Engineering, University of Colorado, Boulder, CO 80309, USA \\ 2. Chemical Engineering, Arizona State University, Tempe, AZ 85287-6106, USA
}

$\triangle$ Corresponding author: Mark Borden, PhD, Assistant Professor, Department of Mechanical Engineering, University of Colorado, 1111 Engineering Drive, Campus Box 427, Boulder, CO 80309, USA. Tel: 303.492.7750; Fax: 303.492.3498; Email: mark.borden@colorado.edu

(C) Ivyspring International Publisher. This is an open-access article distributed under the terms of the Creative Commons License (http://creativecommons.org/ licenses/by-nc-nd/3.0/). Reproduction is permitted for personal, noncommercial use, provided that the article is in whole, unmodified, and properly cited.

Received: 2012.11.09; Accepted: 2012.11.18; Published: 2012.12.10

One of the great engineering contributions to society has been the advent of feedback control to monitor and guide the operation of systems and devices, from maneuvering fighter jets to ensuring the safe operation of petrochemical plants. As healthcare moves toward the personalized medicine paradigm, it is becoming increasingly apparent that real-time feedback control strategies are needed to guide and monitor the delivery of therapies as well. The development of feedback control systems in healthcare will require not just improvements in imaging instrumentation, but also the advent of theranostic agents that can report on and manipulate conditions in vivo. Theranostic biocolloids are needed that can both measure and actuate therapeutic processes. Thus, the field of soft matter colloids is rapidly integrating with the radiological and pharmaceutical fields to add a vast new array of theranostic tools. Theranostic biocolloids are nano- and micro-metric particles which can be injected into the patient for both imaging and therapy. Often, these particles are formed by the directed assembly of biomolecules, imaging tags and active pharmaceutical ingredients, and so a solid foundation in colloid science is necessary.

There appears to be an endless supply of possible combinations of biomolecules (such as lipids, proteins, sugars and nucleic acids) and biocompatible synthetic compounds (such as polymers, dendrimers, fluorocarbons and metal chelators) to form theranostic colloidal constructs. The enormous library of materials and methods available to generate bio- colloids provides unparalleled flexibility; biocolloids can be engineered to carry almost any therapeutic payload and to be detected by any imaging modality. Biocolloids have been engineered as contrast agents for ultrasound, photoacoustic imaging, optical tomography, magnetic resonance imaging (MRI), x-ray computed tomography (CT) and positron emission tomography (PET). Additionally, soft matter colloids have been loaded with pharmaceutical agents to increase their therapeutic index. Combining these functionalities, theranostic biocolloids open the possibility of image-guided therapy, allowing the realization of real-time measurement, monitoring and manipulation of drug targeting.

We are pleased to present this special issue on theranostic biocolloids. We start with several articles on soft nanoparticles. First, Luk et al. review recent work on lipid and polymer nanostructures for cancer theranostics [1]. Menon et al. then present a novel nanoemulsion for dual modality MRI/fluorescence imaging for oximetry [2]. Finally, Vu et al. discuss an innovative theranostic polymer library for transgene delivery to cancer cells [3]. The issue then moves to several articles on theranostic microbubbles. First, Owen et al. describe a fascinating new magnetic microbubble formulation [4]. Kwan et al. then describe a novel theranostic oxygen delivery system [5]. Wrenn et al. review the current state of microbubble acoustical modeling and a novel "nested bubble" theranostic strategy [6]. Matsunaga et al. review recent work on phase-change nanodroplets [7]. Sirsi and Borden re- 
view microbubbles used for gene therapy [8]. Finally, Konofagou provides a timely update on the use of focused ultrasound and microbubbles for targeting drugs through the blood-brain barrier [9]. We hope you enjoy our special issue and look forward to seeing how the field of theranostics continues to develop with the field of soft matter biocolloids.

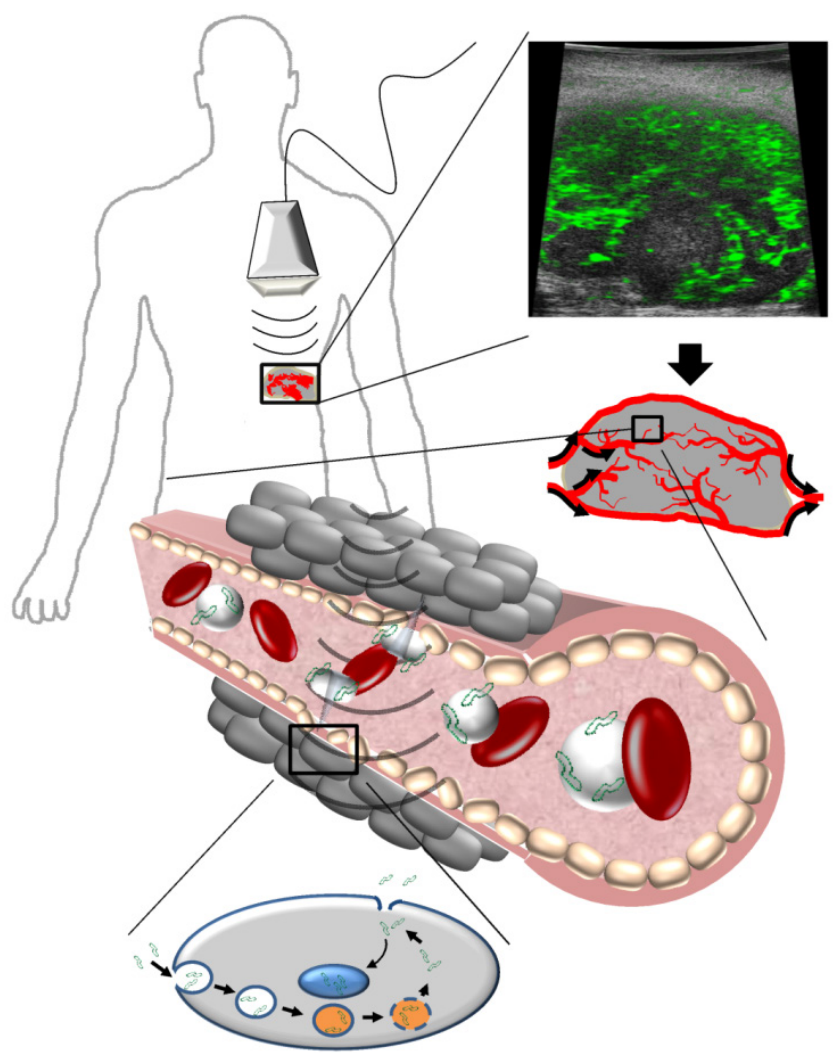

Figure I. Illustration of theranostic microbubbles whose imaging and drug delivery capabilities may be used to implement novel feedback control methods to improve therapy. Created by Shashank Sirsi.

\section{References}

1. Luk BT, Fang RH, Zhang L. Lipid- and polymer-based nanostructures for cancer theranostics. Theranostics 2012; 2(12): 1117-1126. doi: $10.7150 /$ thno.4381.

2. Menon JU, Gulaka PK, McKay MA, Geethanath S, Liu L, Kodibagkar VD. Dual-modality, dual-functional nanoprobes for cellular and molecular imaging. Theranostics 2012; 2(12): 1199-1207. doi:10.7150/thno.4812.

3. Vu L, Ramos J, Potta T, Rege K. Generation of a Focused Poly(amino ether) Library: Polymer-mediated Transgene Delivery and Gold-Nanorod based Theranostic Systems. Theranostics 2012; 2(12):1160-1173.

4. Owen J, Zhou B, Rademeyer P, Tang MX, Pankhurst Q, Eckersley R, Stride E. Understanding the Structure and Mechanism of Formation of a New Magnetic Microbubble Formulation. Theranostics 2012; 2(12):1127-1139.

5. Kwan JJ, Kaya M, Borden MA, Dayton PA. Theranostic Oxygen Delivery Using Ultrasound and Microbubbles. Theranostics 2012; 2(12):1174-1184. doi: $10.7150 /$ thno. 4410 .

6. Wrenn SP, Dicker SM, Small EF, Dan NR, Mleczko M, Schmitz G, Lewin PA. Bursting Bubbles and Bilayers. Theranostics 2012; 2(12):1140-1159.
7. Matsunaga TO, Sheeran PS, Luois S, Streeter JE, Mullin LB, Banerjee B, Dayton PA. Phase-Change Nanoparticles Using Highly Volatile Perfluorocarbons: Toward a Platform for Extravascular Ultrasound Imaging. Theranostics 2012; 2(12):1185-1198. doi:10.7150/thno.4846.

8. Sirsi SR, Borden MA. Advances in Ultrasound Mediated Gene Therapy Using Microbubble Contrast Agents. Theranostics 2012; 2(12):1208-1222. doi:10.7150/thno.4306.

9. Konofagou EE. Optimization of the Ultrasound-Induced Blood-Brain Barrier Opening. Theranostics 2012; 2(12):1223-1237. doi:10.7150/ thno.5576. 